\title{
Validated HPLC Method for Quantification of Pregabalin in Human Plasma Using 1-Fluoro-2,4-dinitrobenzene as Derivatization Agent
}

\author{
Reza Ahmadkhaniha, ${ }^{1}$ Siavash Mottaghi, ${ }^{2}$ Mohammad Zargarpoor, ${ }^{2}$ and Effat Souri ${ }^{2}$ \\ ${ }^{1}$ Department of Human Ecology, School of Public health, Tehran University of Medical Sciences, Tehran, Iran \\ ${ }^{2}$ Department of Medicinal Chemistry, Faculty of Pharmacy and Pharmaceutical Sciences Research Center, \\ Tehran University of Medical Sciences, Tehran 14155-6451, Iran \\ Correspondence should be addressed to Effat Souri; souri@sina.tums.ac.ir
}

Received 29 May 2014; Accepted 14 July 2014; Published 17 August 2014

Academic Editor: Toyohide Takeuchi

Copyright (C) 2014 Reza Ahmadkhaniha et al. This is an open access article distributed under the Creative Commons Attribution License, which permits unrestricted use, distribution, and reproduction in any medium, provided the original work is properly cited.

In this study, a sensitive, simple, and reliable HPLC method for quantification of pregabalin in human plasma was developed and validated. 1-Fluoro-2,4-dinitrobenzene was used as precolumn derivatization agent. For chromatography, an analytical reversed phase (C18) column and a mixture of $\mathrm{Na}_{2} \mathrm{HPO}_{4} 10 \mathrm{mM}(\mathrm{pH} 8.0)$ - methanol (35:65 v/v) were used as stationary and mobile phase, respectively. Detection was performed using a UV detector tuned at $360 \mathrm{~nm}$. The linearity of the method was tested over the concentration range $1-4500 \mathrm{ng} / \mathrm{mL}$ in $500 \mu \mathrm{L}$ of human plasma and satisfactory results were obtained $\left(r^{2}>0.999\right)$. The method showed good precision and accuracy in terms of within - between days relative standard deviations and percent deviation from nominated values (in the range of $4.3-12.7 \%$ and $2.6-8.0 \%$, resp.). The limit of quantification of the method was found to be $1 \mathrm{ng} / \mathrm{mL}$ which is better than previously reported method and indicates its potential application for sensitive bioanalysis.

\section{Introduction}

Pregabalin (Figure 1), (S)-3-(amino ethyl)-5-methylhexanoic acid, an analogue of gamma amino butyric acid (GABA) with lipophilic properties, is a potent agonist of $\alpha_{2} \delta$ subunit of voltage dependent calcium channels [1]. Pregabalin reduces release of glutamate, noradrenalin, substance $\mathrm{P}$, serotonin, and dopamine in central nervous system (CNS) [2-4] and could be used for the treatment of pathological conditions such as partial seizure, neuropathic pain, and generalized anxiety disorder [5-9]. After being taken orally, pregabalin is absorbed rapidly and reaches maximum plasma concentration $\left(C_{\max }\right)$ at about $1.3 \mathrm{~h}[10]$. A range of $\left(C_{\max }\right)$ from 3.5 to $4.5 \mu \mathrm{g} / \mathrm{mL}$ was reported after orally administration of $150 \mathrm{mg}$ pregabalin to the volunteers [11].

For determination of pregabalin in biological fluids, sophisticated methods such as methods based on LC-MSMS were employed [11-14]. Although most of LC-MS-MS methods are sensitive and reliable, the instruments are too expensive and unavailable in most of clinical laboratories. Furthermore the carry-over and ion suppression effects are main analytical problems of LC-MS methods which are against the routine use of these methods $[15,16]$.

Pregabalin is an aliphatic agent without any significant chromophore group, which makes difficulty in its quantification by general HPLC-UV methods. Therefore, derivatizing reagents such as o-phthaldialdehyde (OPA), 3mercaptopropionic acid, and picrylsulfonic acid were usually used to make better determination $[10,17,18]$. Unfortunately, most of these derivatization methods are complicated and time consuming and suffer from low recovery values. Based on our knowledge, there is only one report about liquid chromatographic analysis of pregabalin in human urine without derivatization [19]. However, the method sensitivity was not adequate for most of bioanalytical application such as pharmacokinetic studies. Pharmacokinetic studies require 


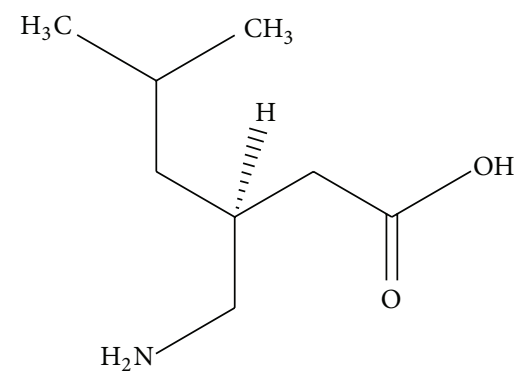

FIGURE 1: Chemical structure of pregabalin.<smiles>O=[N+]([O-])c1ccc(F)c([N+](=O)[O-])c1</smiles>

Figure 2: Chemical structure of FDNB.

a simple, sensitive, selective, reliable, and rapid analytical method. In this study, a nucleophilic reagent, 1-fluoro-2,4dinitrobenzene (FDNB) (Figure 2), was used for production of a chromophoric derivative of pregabalin. This reagent has been used before for the determination of pregabalin in bulk raw materials and also in pharmaceutical dosage forms [2023]. However, based on our knowledge, there is no report about application of this derivatization agent for pregabalin bioanalysis. The purpose of our study was to develop a rapid, sensitive, and reliable bioanalytical method based on precolumn derivatization for analysis of pregabalin in human plasma.

\section{Experimental}

2.1. Chemicals. Pregabalin was from Sun Pharma (India) and was kindly provided by Bakhtar Bioshimi Co. (Kermanshah, Iran). Gabapentin (Internal Standard, I.S.) was from Ranbaxy (India). 1-Fluoro-2,4-dinitrobenzene (FDNB) and all other solvents and regents were of analytical grade and purchased from Merck (Darmstadt, Germany). The drug-free plasma of healthy volunteers was provided by the Blood Centre of Shariatee University Hospital (Tehran, Iran). HPLC grade water was prepared using a Milli-Q purification system (Millipore, Milford, MA, USA).

2.2. Instrumentation. An Agilent 1100 system (Agilent Technologies, Waldbronn, Germany) consisting of a degasser, a quaternary pump, a column oven, an auto sampler, and a variable wavelength UV detector was used for separation and determination of the target analytes.

2.3. Standard Solutions. The stock standard solutions of pregabalin and gabapentin (I.S.) were prepared at $25 \mu \mathrm{g} / \mathrm{mL}$ in methanol. Calibration solutions of pregabalin were prepared by stepwise dilution using methanol as diluent. Stock solution of FDNB $(0.06 \mathrm{M})$ was prepared by dissolving $1.14 \mathrm{~g}$ of the reagent in $100 \mathrm{~mL}$ of acetonitrile (this reagent should be handled carefully since it is a skin irritant). Appropriate amounts of $\mathrm{H}_{3} \mathrm{BO}_{3}$ and $\mathrm{KCl}$ were dissolved in water and the $\mathrm{pH}$ was adjusted to 8.2 by adding $2 \mathrm{M} \mathrm{NaOH}$ to prepare a $0.25 \mathrm{M}$ borate buffer. All stock and working solutions were stored at $4^{\circ} \mathrm{C}$ until analysis.

2.4. Sample Preparation. To $500 \mu \mathrm{L}$ of drug-free plasma, $10 \mu \mathrm{L}$ of internal standard solution $(25 \mu \mathrm{g} / \mathrm{mL})$, and $10 \mu \mathrm{L}$ of calibration standard solutions were added and the mixture was vortex-mixed for $10 \mathrm{~s}$. After addition of $1 \mathrm{~mL}$ acetonitrile to the samples, the tubes were shaken for another $10 \mathrm{~s}$. After centrifugation at $4472 \times \mathrm{g}\left(4^{\circ} \mathrm{C}, 5 \mathrm{~min}\right)$, the upper layer was transferred into another vial containing borate buffer $(100 \mu \mathrm{L})$ and FDNB solution $(50 \mu \mathrm{L})$. The mixture was vortex-mixed for $10 \mathrm{~s}$ and kept at $65^{\circ} \mathrm{C}$ for $20 \mathrm{~min}$. The samples was cooled to room temperature and after addition of saturated $\mathrm{NaCl}$ solution $(100 \mu \mathrm{L})$ and $n$-pentane $(100 \mu \mathrm{L})$, the mixture was shaken for $30 \mathrm{~s}$. After centrifugation at $1610 \times \mathrm{g}$ for $5 \mathrm{~min}$, the lower layer was transferred into vials containing $250 \mu \mathrm{L}$ of $\mathrm{HCl}(3 \mathrm{M})$ and $500 \mu \mathrm{L}$ of chloroform. The mixture was shaken for $1 \mathrm{~min}$ and centrifuged at $1610 \times \mathrm{g}$ for $5 \mathrm{~min}$ and the chloroform layer was transferred into another vial and evaporated at $50^{\circ} \mathrm{C}$ under nitrogen stream. The residue was reconstituted in $50 \mu \mathrm{L}$ of methanol, vortex-mixing for $10 \mathrm{~s}$ and an aliquot of $20 \mu \mathrm{L}$ was injected onto the HPLC system for analysis.

2.5. Chromatographic Conditions. Chromatographic separation was achieved on a reversed-phase TRACER EXCEL ODS-A stainless steel column, $(5 \mu \mathrm{m}, 150 \times 4.6 \mathrm{~mm}$ i.d., Teknokroma, Barcelona, Spain) at ambient temperature. Mobile phase consisted of methanol (65\%) and $\mathrm{Na}_{2} \mathrm{HPO}_{4}$ $(35 \%, \mathrm{pH}=8,0.01 \mathrm{M})$. The flow rate was set at $1.0 \mathrm{~mL} / \mathrm{min}$. Detection was performed at $360 \mathrm{~nm}$.

2.6. Calibration. Standard calibration solutions in the range of $1-5000 \mathrm{ng} / \mathrm{mL}$ were prepared by spiking blank plasma samples with appropriate amounts of gabapentin $(10 \mu \mathrm{L} \times$ $25 \mu \mathrm{g} / \mathrm{mL}$ ) as internal standard and pregabalin calibration solutions to produce final concentrations equivalent to 1 , $10,25,100,250,500,1000,2000,4000$, and $5000 \mathrm{ng} / \mathrm{mL}$ of pregabalin. Calibration samples were prepared in five replicates and the results (ratio of peak areas versus nominal concentration) were used to compute the calibration equation. The quality of linear regression analysis was evaluated by comparing the results of determined concentrations to the nominal ones.

2.7. Quality Control Samples. The quality control samples (QC) were prepared in the same way as the calibration samples at different concentrations along the calibration range $(1,5,75,450,550,2500$, and $4500 \mathrm{ng} / \mathrm{mL})$. The calibration and quality control samples were under the same storage and analytical conditions. 
2.8. Validation of the Method. The method was validated for linearity, accuracy, and precision using FDA guidelines [24]. Precision (expressed as \% relative standard deviation, R.S.D) and accuracy (expressed as \% error) were calculated for QC samples. Five replicates at each QC level were analyzed to determine the intraday accuracy and precision. This process was repeated over three days in order to determine the interday accuracy and precision. The extraction recoveries were determined by comparing the results of quality control samples with standard solutions at the same nominal concentrations.

2.9. Pharmacokinetic Study. The developed method was used for the determination of pregabalin concentration in human plasma after administration of a single oral dose of pregabalin (Parke-Davis, Germany, Lot number 0076125) to two healthy adult male volunteers. The subjects were informed about the aim, course, and possible risks of the study and then gave written consent to their participation. The protocol of study was reviewed and approved by the Ethical Review Committee of Tehran University of Medical Sciences in accordance with the principles of the Declaration of Helsinki and the recommendations of the U.S. Food and Drug Administration. After an overnight fast, each subject received $400 \mathrm{mg}$ of gabapentin in the form of tablet. Blood samples $(5 \mathrm{~mL})$ were drawn into heparinized test tubes immediately before $(0 \mathrm{~h})$ and at $0.5,1,1.5,2,2.5,3,4,5,6,8,10,12$, and $24 \mathrm{~h}$ after drug administration. Blood samples were centrifuged at $2000 \mathrm{~g}$ for $10 \mathrm{~min}$ and plasma samples were separated and stored at $-20^{\circ} \mathrm{C}$ until analysis. Before analysis, the plasma samples were thawed at $18^{\circ} \mathrm{C}$.

\section{Results and Discussion}

3.1. Derivatization Reaction. Reaction of FDNB with primary amines is usually carried out in mild basic medium. On the other hand, hydrolysis of the reagent occurs in alkaline conditions too [22]. The optimum condition for derivatization reaction was selected based on our previous study on gabapentin [23]. The time for reaction was exceeded to $20 \mathrm{~min}$ for achieving better results. The maximum UV absorption of the pregabalin derivative was determined at $360 \mathrm{~nm}$ using wavelength scan experiments in the range of 200-400 nm. A representative UV spectrum of synthesized pregabalin derivative was demonstrated in Figure 3. As indicated, the derivative shows absorption maxima at $360 \mathrm{~nm}$ whereas this absorption peak is absent in the parent compound (pregabalin).

3.2. Chromatography. The chromatographic conditions, especially the analytical column and the composition of mobile phase, were optimized through several trials to achieve the desired sensitivity, separation, run time, and peak shapes. Typical chromatograms obtained for blank plasma, a spiked plasma sample, and a real sample after drug administration are presented in Figure 4. Under described chromatographic conditions, pregabalin and I.S. were well resolved and eluted at about 4.5 and $5.2 \mathrm{~min}$, respectively. No interferences from

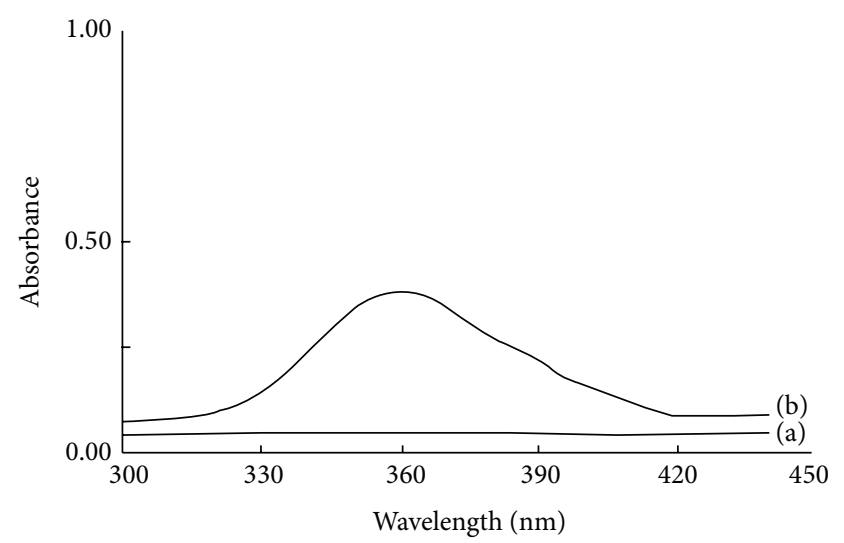

FIGURE 3: A representative UV spectrum of pregabalin (a) and pregabalin-FDNB derivative (b).

TABLE 1: Statistical data of calibration curves of pregabalin in standard solutions $(n=5)$.

\begin{tabular}{lc}
\hline Parameters & Results \\
\hline Linearity range & $1-5000(\mathrm{ng} / \mathrm{mL})$ \\
Regression equation & $Y=0.025 X+0.002$ \\
Standard deviation of slope & 0.13 \\
Relative standard deviation of slope (\%) & 0.72 \\
Standard deviation of intercept & 0.25 \\
Correlation coefficient $\left(r^{2}\right)$ & 0.99 \\
\hline
\end{tabular}

endogenous plasma components or derivatizing reagent were observed.

3.3. Recovery and Analyte Stability. We evaluated a twostep liquid-liquid extraction method for sample preparation. As the results indicated, the extraction method produced a cleaner baseline and consequently higher sensitivity than previously reported one-step liquid-liquid extraction method [23]. In the first step of extraction, the basic condition $(\mathrm{pH}>$ 9.5), results in the conversion of carboxylic acid moiety of the analytes to carboxylate anion, consequently about 95\% (as estimated in preliminary studies) of the analytes will remain in the aqueous phase. After decreasing $\mathrm{pH}$ value $(\mathrm{pH}<2)$ in the second step, the carboxylate anion converts to the corresponding carboxylic acid, which makes the compound only slightly soluble in the aqueous phase. Consequently, considerable amount of the analyte will be extracted by the organic phase. On the other hand, in acidic medium, the colored byproduct of the reaction converts to uncolored compound which results in a cleaner chromatogram. Chloroform was selected as extraction solvent in the second step due to its highest extraction yield. The mean recovery was estimated $88.5 \%$ with RSD value of $3.8 \%$. Pregabalin was found to be stable in human plasma at $-20^{\circ} \mathrm{C}$ for 60 days. The FDNB derivative of pregabalin was also found stable for at least $24 \mathrm{~h}$ at room temperature.

3.4. Validation. A good linear relationship was found between the peak area ratios (pregabalin derivative to that of the 


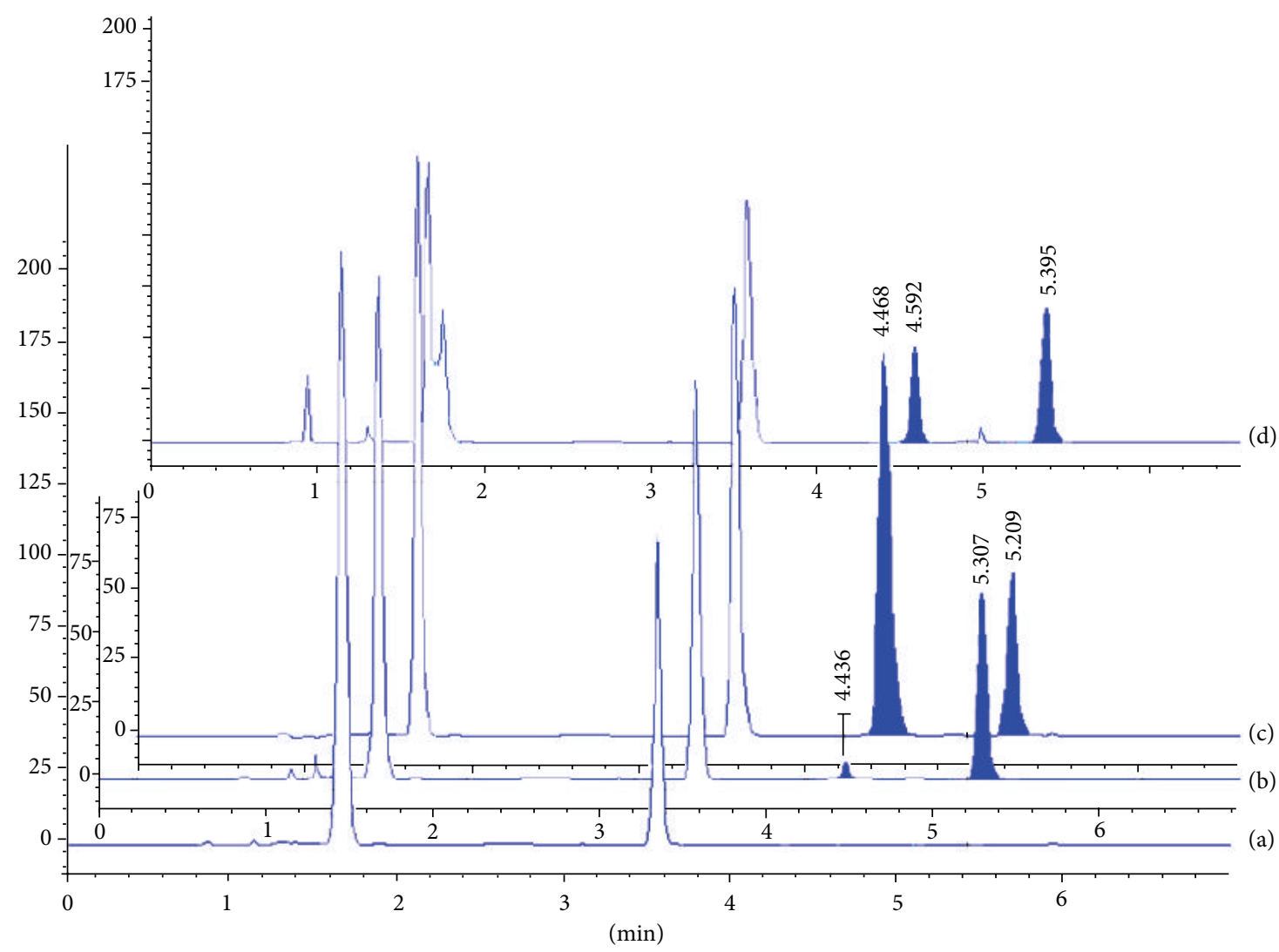

FIGURE 4: Typical HPLC chromatograms of pregabalin and I.S. derivative. (a) drug free plasma; (b) plasma spiked with pregabalin (5 ng/mL) and I.S.; (c) plasma spiked with pregabalin (100 ng/mL) and I.S.; (d) a real sample (concentration of pregabalin was determined as $33 \mathrm{ng} / \mathrm{mL}$ ).

TABLE 2: Precision and accuracy of method for determination of pregabalin in standard solutions ( $n=15$; five sets for 3 days).

\begin{tabular}{|c|c|c|c|c|c|c|}
\hline \multirow{2}{*}{ Nominal concentration $(\mathrm{ng} / \mathrm{mL})$} & \multicolumn{3}{|c|}{ Intraday $(n=5)$} & \multicolumn{3}{|c|}{ Interday $(n=15)$} \\
\hline & Determined $(\mathrm{ng} / \mathrm{mL})$ & RSD (\%) & Error $(\%)$ & Determined $(\mathrm{ng} / \mathrm{mL})$ & RSD (\%) & Error (\%) \\
\hline 1.00 & 1.07 & 10.5 & 7.0 & 1.08 & 12.7 & 8.0 \\
\hline 5.00 & 5.17 & 8.1 & 3.4 & 5.23 & 10.7 & 4.6 \\
\hline 75.00 & 78.10 & 6.2 & 4.1 & 70.33 & 10.1 & -6.2 \\
\hline 450.00 & 438.40 & 4.3 & -2.6 & 413.87 & 4.7 & -8.0 \\
\hline 550.00 & 528.50 & 6.4 & -3.9 & 511.17 & 8.3 & -7.1 \\
\hline 2500.00 & 2419.53 & 8.6 & -3.2 & 2404.67 & 8.9 & -3.8 \\
\hline 4500.00 & 4679.77 & 10.2 & 4.0 & 4325.10 & 10.4 & -3.9 \\
\hline
\end{tabular}

internal standard derivative) versus the corresponding concentration over the range of $1-5000 \mathrm{ng} / \mathrm{mL}$. The calibration curve equation and the regression analysis data are presented in Table 1. The accuracy and precision of the proposed method were determined at different concentrations. Intraday and interday experiment data are presented in Table 2. RSD and error values, respectively, $<12.7 \%$ and $<8.0 \%$, indicate adequate accuracy and precision of the method. Limit of quantification was found to be $1 \mathrm{ng} \mathrm{mL}^{-1}(\mathrm{RSD}<12.7 \%)$ for pregabalin.

3.5. Application to Pharmacokinetic Study. The average plasma concentration-time profile of pregabalin after administration of a single oral dose of pregabalin tablet (400 mg) to two volunteers is shown in Figure 5. As indicated in this graph, pregabalin has been quantified more than 5 elimination half-lives after $C_{\max }$ which shows the method applicability in pharmacokinetic studies. Under the above mentioned experimental condition, the main pharmacokinetic parameters were determined as $C_{\max }=8.2 \mu \mathrm{g} / \mathrm{mL}, T_{\max }$ $=2-3 \mathrm{~h}$, and mean elimination half time $\left(T_{1 / 2}\right)=6.3 \mathrm{~h}$.

\section{Conclusion}

In this study, a simple HPLC method for quantification of pregabalin in human plasma was developed. The validated method is based on the precolumn derivatization of pregabalin with FDNB in alkaline medium. The colored derivative 
TABLE 3: A brief comparison between different HPLC methods for determination of pregabalin in human plasma.

\begin{tabular}{|c|c|c|c|c|c|}
\hline Reference & Derivatizing reagent & Linearity range $(\mu \mathrm{g} / \mathrm{mL})$ & Detection & Retention time (min) & $\mathrm{LOQ}(\mu \mathrm{g} / \mathrm{mL})$ \\
\hline$[15]$ & Picrylsulfonic acid & $0.5-8$ & Fluorescence & 17.0 & 0.25 \\
\hline$[16]$ & o-Phthaldialdehyde & $0-40$ & Fluorescence & 15.0 & 0.13 \\
\hline$[24]$ & 4-Chloro-7-nitrobenzofuran & $0.4-30$ & Fluorescence & 14.0 & 0.4 \\
\hline $\begin{array}{l}\text { The method developed in } \\
\text { this study }\end{array}$ & 1-Fluoro-2,4-dinitrobenzene & $1-5000$ & UV & 5.3 & 0.001 \\
\hline
\end{tabular}

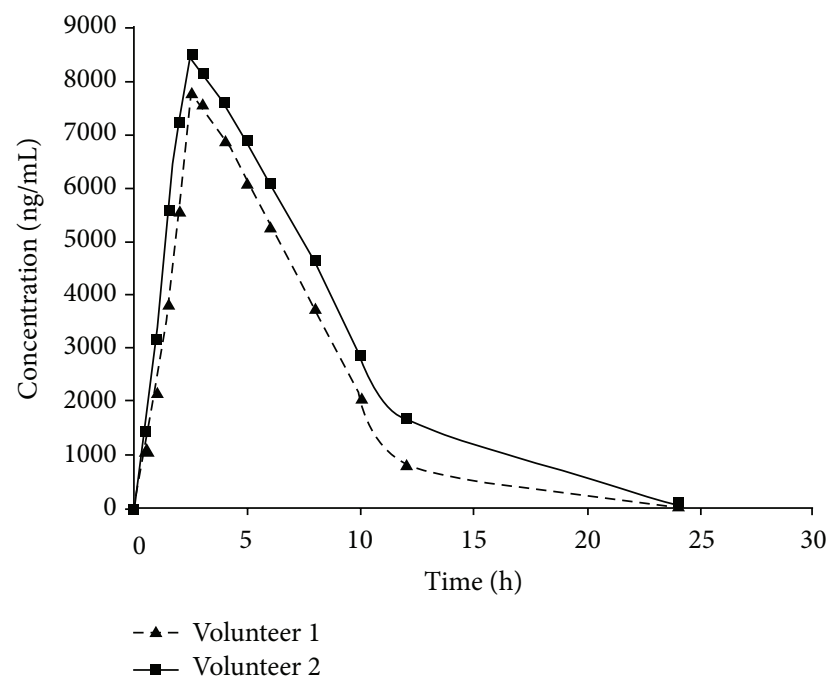

FIGURE 5: Plasma concentration-time profile of pregabalin after administration of a single dose $(400 \mathrm{mg})$ pregabalin to two volunteers.

could be determined by general UV detector even at low concentration. A total analysis time (including both sample preparation and instrument run time) of less than $60 \mathrm{~min}$ makes this method suitable for high-throughput analysis. Based on our literature review, the best reported limit of quantification for HPLC determination of pregabalin in plasma was $0.13 \mu \mathrm{g} / \mathrm{mL}[15,16,25]$. The limit of quantification of the present method is better than these methods and is comparable to the previously LC-MS-MS reported methods [11-14] (Table 3).

\section{Conflict of Interests}

The authors declare that there is no conflict of interests regarding the publication of this paper.

\section{References}

[1] M. A. Rogawski and C. P. Taylor, "Calcium channel $\alpha_{2}-\delta$ subunit, a new antiepileptic drug target," Epilepsy Research, vol. 69, no. 3, pp. 183-272, 2006.

[2] D. J. Dooley, C. M. Donovan, and T. A. Pugsley, "Stimulusdependent modulation of $\left[{ }^{3} \mathrm{H}\right]$ norepinephrine release from rat neocortical slices by gabapentin and pregabalin," Journal of Pharmacology and Experimental Therapeutics, vol. 295, no. 3, pp. 1086-1093, 2000.
[3] D. J. Dooley, C. A. Mieske, and S. A. Borosky, "Inhibition of $\mathrm{K}^{+}$evoked glutamate release from rat neocortical and hippocampal slices by gabapentin," Neuroscience Letters, vol. 280, no. 2, pp. 107-110, 2000.

[4] K. Fink, D. J. Dooley, W. P. Meder et al., "Inhibition of neuronal $\mathrm{Ca}^{2+}$ influx by gabapentin and pregabalin in the human neocortex," Neuropharmacology, vol. 42, no. 2, pp. 229236, 2002.

[5] G. Zareba, "New treatment options in the management of fibromyalgia: role of pregabalin," Neuropsychiatric Disease and Treatment, vol. 4, no. 6, pp. 1193-1201, 2008.

[6] J. E. Frampton and R. H. Foster, "Pregabalin,” Drugs, vol. 65, no. 1, pp. 111-118, 2005.

[7] P. Gray, "Pregabalin in the management of central neuropathic pain," Expert Opinion on Pharmacotherapy, vol. 8, no. 17, pp. 3035-3041, 2007.

[8] G. Zareba, "Pregabalin: a new agent for the treatment of neuropathic pain," Drugs of Today, vol. 41, no. 8, pp. 509-516, 2005.

[9] B. F. Shneker and J. W. McAuley, "Pregabalin: a new neuromodulator with broad therapeutic indications," Annals of Pharmacotherapy, vol. 39, no. 12, pp. 2029-2037, 2005.

[10] H. N. Bockbrader, L. L. Radulovic, E. L. Posvar et al., "Clinical pharmacokinetics of pregabalin in healthy volunteers," The Journal of Clinical Pharmacology, vol. 50, no. 8, pp. 941-950, 2010.

[11] V. V. Vaidya, S. M. Yetal, S. M. N. Roy, N. A. Gomes, and S. S. Joshi, "LC-MS-MS determination of pregabalin in human plasma," Chromatographia, vol. 66, no. 11-12, pp. 925-928, 2007.

[12] U. Mandal, A. K. Sarkar, K. V. Gowda et al., "Determination of pregabalin in human plasma using LC-MS-MS," Chromatographia, vol. 67, no. 3-4, pp. 237-243, 2008.

[13] R. Nirogi, V. Kandikere, K. Mudigonda, P. Komarneni, and R. Aleti, "Liquid chromatography atmospheric pressure chemical ionization tandem mass spectrometry method for the quantification of pregabalin in human plasma," Journal of Chromatography B: Analytical Technologies in the Biomedical and Life Sciences, vol. 877, no. 30, pp. 3899-3906, 2009.

[14] G. R. Shah, C. Ghosh, and B. T. Thaker, "Determination of pregabalin in human plasma by electrospray ionisation tandem mass spectroscopy", Journal of Advanced Pharmaceutical Technology and Research, vol. 1, no. 3, pp. 354-357, 2010.

[15] N. Rastkari, M. Khoobi, A. Shafiee, M. R. Khoshayand, and R. Ahmadkhaniha, "Development and validation of a simple and sensitive HPLC-UV method for the determination of captopril in human plasma using a new derivatizing reagent 2-naphthyl propiolate," Journal of Chromatography B, vol. 932, pp. 144-151, 2013.

[16] N. Rastkari and R. Ahmadkhaniha, "Magnetic solid-phase extraction based on magnetic multi-walled carbon nanotubes 
for the determination of phthalate monoesters in urine samples," Journal of Chromatography A, vol. 1286, pp. 22-28, 2013.

[17] D. Berry and C. Millington, "Analysis of pregabalin at therapeutic concentrations in human plasma/serum by reversed-phase HPLC," Therapeutic Drug Monitoring, vol. 27, no. 4, pp. 451-456, 2005.

[18] T. A. C. Vermeij and P. Edelbroek, "Simultaneous highperformance liquid chromatographic analysis of pregabalin, gabapentin and vigabatrin in human serum by precolumn derivatization with o-phtaldialdehyde and fluorescence detection," Journal of Chromatography B: Analytical Technologies in the Biomedical and Life Sciences, vol. 810, no. 2, pp. 297-303, 2004.

[19] R. S. Gujral, S. M. Haque, and S. Kumar, "A novel method for the determination of pregabalin in bulk pharmaceutical formulations and human urine samples," African Journal of Pharmacy and Pharmacology, vol. 3, no. 6, pp. 327-334, 2009.

[20] T. Wu, Q. Jing, B. Ye, and Y. Shen, "Determination of Pregabalin by HPLC with precolumn derivatization using 1-fluoro-2,4dinitrobenzene," Journal of Chemical Engineering of Chinese Universities, vol. 2, pp. 350-353, 2010.

[21] E. Souri, M. Eskandari, M. Barazandeh Tehrani, N. Adib, and R. Ahmadkhaniha, "HPLC determination of pregabalin in bulk and pharmaceutical dosage forms after derivatization with 1fluoro-2,4-dinitrobenzene," Asian Journal of Chemistry, vol. 25, no. 13, pp. 7332-7336, 2013.

[22] R. A.-A. Shaalan, "Spectrofluorimetric and spectrophotometric determination of pregabalin in capsules and urine samples," International Journal of Biomedical Science, vol. 6, no. 3, pp. 260267, 2010.

[23] H. Jalalizadeh, E. Souri, M. B. Tehrani, and A. Jahangiri, "Validated HPLC method for the determination of gabapentin in human plasma using pre-column derivatization with 1-fluoro2,4-dinitrobenzene and its application to a pharmacokinetic study," Journal of Chromatography B, vol. 854, no. 1-2, pp. 4347, 2007.

[24] F. Garofolo, "Bioanalytical method validation," in Analytical Method Validation and Instrument Performance Verification, chapter 8, pp. 105-138, Wiley-Interscience, Hoboken, NJ, USA, 2004.

[25] B. Martinc, R. Roskar, I. Grabnar, and T. Vovk, "Simultaneous determination of gabapentin, pregabalin, vigabatrin, and topiramate in plasma by HPLC with fluorescence detection," Journal of Chromatography B, vol. 962, pp. 82-88, 2014. 

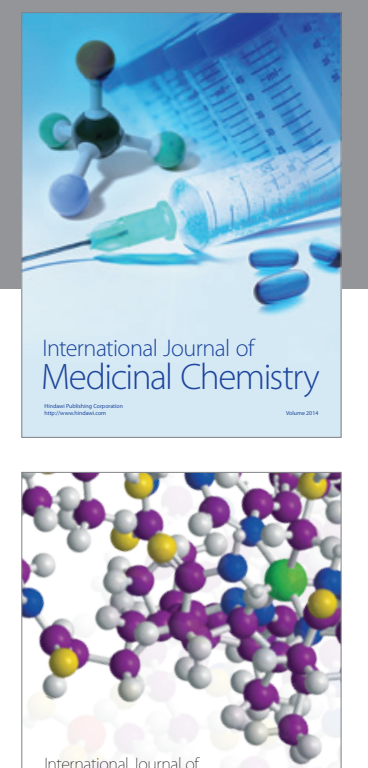

\section{Carbohydrate} Chemistry

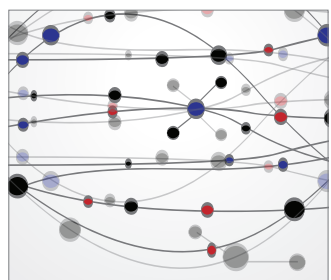

The Scientific World Journal
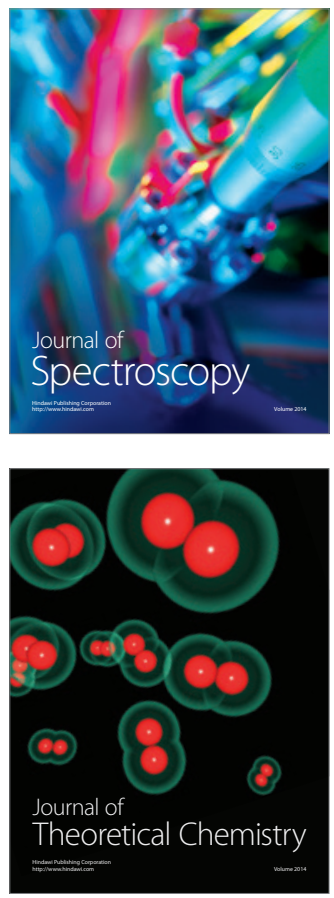
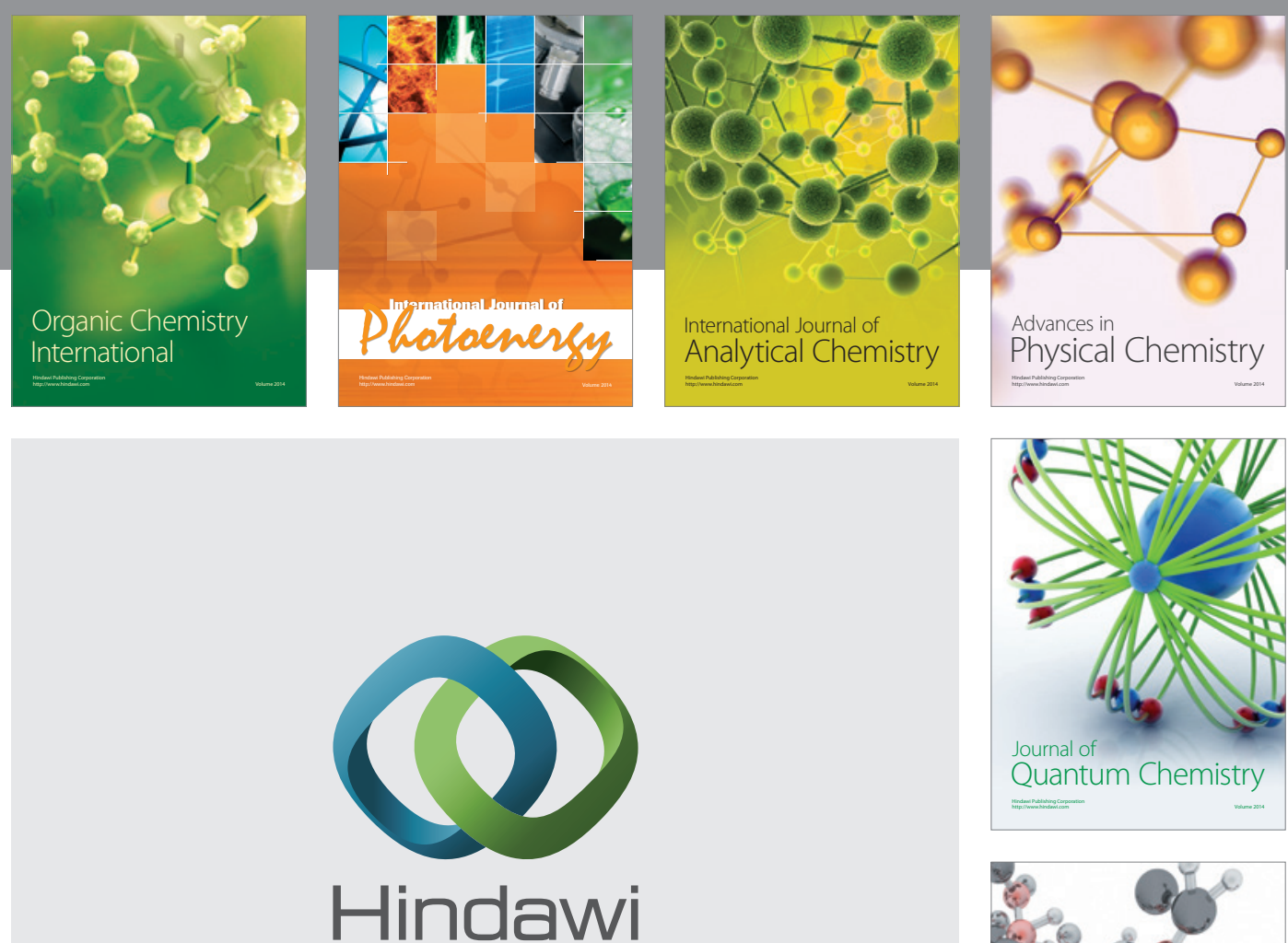

Submit your manuscripts at

http://www.hindawi.com

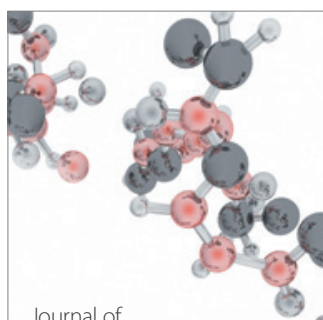

Analytical Methods

in Chemistry

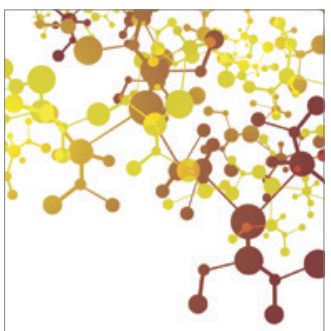

Journal of

Applied Chemistry

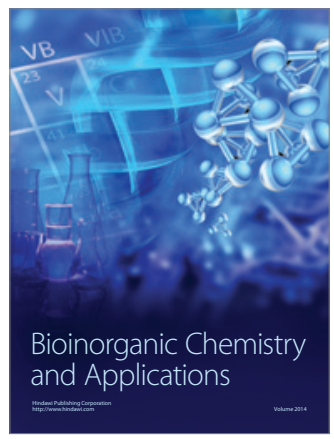

Inorganic Chemistry
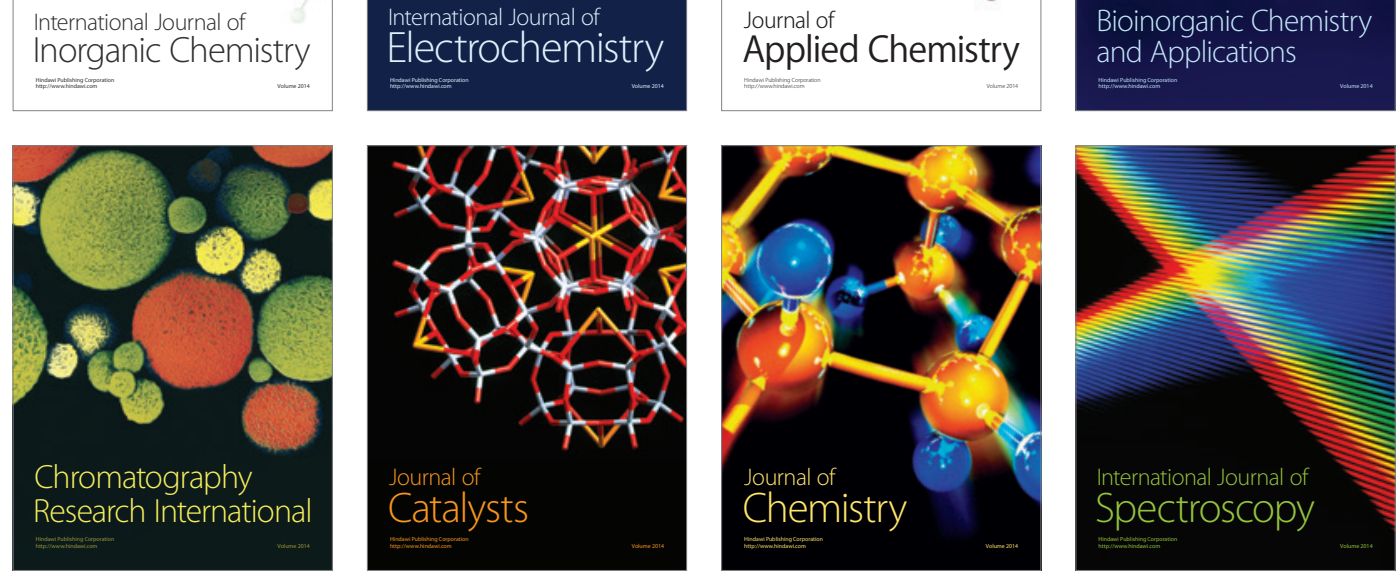